\title{
Almost Jordan homomorphisms and Jordan derivations associated to the parametric-additive functional equation on fuzzy Banach algebras
}

\author{
Hassan Azadi Kenary ${ }^{1}$, Hamid Rezaei ${ }^{1}$, Yosouf Gheisari ${ }^{2}$, Madjid Eshaghi Gordji ${ }^{3}$ and Dong Yun Shin ${ }^{4 *}$
}

* Correspondence: dyshin@uos.ac.

${ }^{4}$ Department of Mathematics, College of Natural Science, University of Seoul, Seoul 130-743, Korea

Full list of author information is available at the end of the article

\section{Abstract}

In this article, we establish the generalized Hyers-Ulam (or Hyers-Ulam-Rassais) stability of Jordan homomorphisms and Jordan derivations of the following parametric additive functional equation:

$$
\sum_{i=1}^{m} f\left(x_{i}\right)=\frac{1}{2 m}\left[\sum_{i=1}^{m} f\left(m x_{i}+\sum_{j=1, j \neq i}^{m} x_{j}\right)+f\left(\sum_{i=1}^{m} x_{i}\right)\right]
$$

for a fixed positive integer $m$ with $m \geq 2$, on fuzzy Banach algebras. The concept of Ulam-Hyers-Rassias stability originated from Rassias stability theorem that appeared in his article.

Mathematics Subject Classification: Primary, 46S40; Secondary, 39B52; 39B82; 26E50; 46S50; 46H25.

Keywords: Fuzzy normed space, Jordan homomorphism, Jordan derivation, generalized Hyers-Ulam stability

\section{Introduction}

The stability problem of functional equations originated from a question of Ulam [1] in 1940, concerning the stability of group homomorphisms. Let $\left(G_{1}\right.$, .) be a group and let $\left(G_{2},{ }^{*}\right)$ be a metric group with the metric $d(.$, .). Given $\varepsilon>0$, does there exist a $\delta 0$, such that if a mapping $h: G_{1} \rightarrow G_{2}$ satisfies the inequality $d(h(x . y), h(x) * h(y))<\delta$ for all $x$, $y \in G_{1}$, then there exists a homomorphism $H: G_{1} \rightarrow G_{2}$ with $d(h(x), H(x))<\varepsilon$ for all $x$ $\in G_{1}$ ? In the other words, under what condition does there exist a homomorphism near an approximate homomorphism? The concept of stability for functional equation arises when we replace the functional equation by an inequality which acts as a perturbation of the equation. In 1941, Hyers [2] gave the first affirmative answer to the question of Ulam for Banach spaces. Let $f: E \rightarrow E$ ” be a mapping between Banach spaces such that $\|f(x+y)-f(x)-f(y)\| \leq \delta$ for all $x, y E$, and for some $\delta>0$. Then there exists a unique additive mapping $T: E \rightarrow E^{\prime}$ such that $\|f(x)-T(x)\| \leq \delta$ for all $x \in E$. Moreover if $f(t x)$ is continuous in $t \in \mathbb{R}$ for each fixed $x \in E$, then $T$ is linear. In 1978, Rassias [3] proved the following theorem. 
Theorem 1.1. Let $f: E \rightarrow E^{\prime}$ be a mapping from a normed vector space $E$ into a Banach space $E^{\prime}$ subject to the inequality

$$
\|f(x+y)-f(x)-f(y)\| \leq \epsilon\left(\|x\|^{p}+\|y\|^{p}\right)
$$

for all $x, y \in E$, where and $p$ are constants with $\varepsilon>0$ and $p<1$. Then there exists a unique additive mapping $T: E \rightarrow E$ such that

$$
\|f(x)-T(x)\| \leq \frac{2 \epsilon}{2-2^{p}}\|x\|^{p}
$$

for all $x \in E$. If $p<0$ then inequality (1.1) holds for all $x, y \neq 0$, and (1.2) for $x \neq 0$. Also, if the function $t \mapsto f(t x)$ from $\mathbb{R}$ into $E^{\prime}$ is continuous in real $t$ for each fixed $x \in E$, then $T$ is linear.

In 1991, Gajda [4] answered the question for the case $p>1$, which was raised by Rassias. This new concept is known as the generalized Hyers-Ulam stability of functional equations.

Following [5], we give the employing notion of a fuzzy norm.

Let $\mathrm{X}$ be a real linear space. A function $N: X \times \mathbb{R} \rightarrow[0,1]$ (the so-called fuzzy subset) is said to be a fuzzy norm on $\mathrm{X}$ if for all $x, y \in X$ and all $a, b \in \mathbb{R}$ :

$\left(N_{1}\right) N(x, a)=0$ for $a \leq 0$;

$\left(N_{2}\right) x=0$ if and only if $N(x, a)=1$ for all $a>0$;

$\left(N_{3}\right) N(a x, b)=N\left(x, \frac{b}{|a|}\right)$ if $a \neq 0$;

$\left(N_{4}\right) N(x+y, a+b) \geq \min \{N(x, a), N(y, b)\}$

$\left(N_{5}\right) N(x,$.$) is non-decreasing function on \mathbb{R}$ and $\lim _{a \rightarrow \infty} N(x, a)=1$;

$\left(N_{6}\right)$ For $x \neq 0, N(x,$.$) is (upper semi) continuous on \mathbb{R}$.

The pair $(X, N)$ is called a fuzzy normed linear space. One may regard $N(x, a)$ as the truth value of the statement "the norm of $x$ is less than or equal to the real number $a^{\text {". }}$.

Example 1.2. Let $(X,\|\|$.$) be a normed linear space. Then$

$$
N(x, a)= \begin{cases}\frac{a}{a+\|x\|}, & a>0, x \in X \\ 0, & a \leq 0, x \in X\end{cases}
$$

is a fuzzy norm on $X$.

Let $(X, N)$ be a fuzzy normed linear space. Let $\left\{x_{n}\right\}$ be a sequence in $X$. Then $\left\{x_{n}\right\}$ is said to be convergent if there exists $x \in X$ such that $\lim _{n \rightarrow \infty} N\left(x_{n}-x, a\right)=1$ for all $a>0$. In that case, $x$ is called the limit of the sequence $\left\{x_{n}\right\}$ and we denote it by $N-\lim _{n \rightarrow \infty} x_{n}=x$. A sequence $\left\{x_{n}\right\}$ in $\mathrm{X}$ is called Cauchy if for each $\varepsilon>0$ and each $a$ there exists $n_{0}$ such that for all $n \geq n_{0}$ and all $p>0$, we have $N\left(x_{n+p}-\mathrm{x}_{\mathrm{n}}, a\right)>1-\varepsilon$. It is known that every convergent sequence in fuzzy normed space is Cauchy. If each Cauchy sequence is convergent, then the fuzzy norm is said to be complete and the fuzzy normed space is called a fuzzy Banach space.

Let $X$ be an algebra and $(X, N)$ be complete fuzzy normed space, the pair $(X, N)$ is said to be a fuzzy Banach algebra if for every $x, y \in X, a, b \in \mathbb{R}$

$$
N(x y, a b) \geq \max \{N(x, a), N(y, b)\} .
$$

Let $(X, N)$ be a fuzzy Banach algebra and $\left\{x_{n}\right\},\left\{y_{n}\right\}$ be convergent sequences in $(X, N)$ such that $N-\lim _{n \rightarrow \infty} x_{n}=x$ and $N-\lim _{n \rightarrow \infty} y_{n}=y$. Then

$$
\begin{aligned}
N\left(x_{n} y_{n}-x y, 2 t\right) & \geq \min \left\{N\left(\left(x_{n}-x\right) y_{n}, t\right), N\left(x\left(y_{n}-y\right), t\right)\right\} \\
& \geq \min \left\{N\left(x_{n}-x, t\right), N\left(y_{n}-y, t\right)\right\}
\end{aligned}
$$


for all $t>0$. Therefore $N-\lim _{n \rightarrow \infty} x_{n} y_{n}=x y$.

The generalized Hyers-Ulam stability of different functional equations in random normed and fuzzy normed spaces has been recently studied in [6-9].

Definition 1.3. Suppose $A$ and $B$ are two Banach algebras. We say that a mapping $h$ : $A \rightarrow B$ is a Jordan homomorphism if

$$
h(a+b)=h(a)+h(b), \quad \text { and } \quad h\left(a^{2}\right)=h(a)^{2}
$$

for all $a, b \in A$.

Definition 1.4. Suppose $A$ is a Banach algebra. We say that a mapping $d: A \rightarrow A$ is a Jordan derivation if

$$
d(a+b)=d(a)+d(b), \quad \text { and } d\left(a^{2}\right)=a d(a)+d(a) a
$$

for all $a, b \in A$.

The stability of different functional equations in various normed spaces and also on Banach algebras has been recently studied in [2-4,6-29].

In the present article, we investigate the generalized Hyers-Ulam stability of Jordan homomorphisms and Jordan derivations of the following parametric-additive functional equation

$$
\sum_{i=1}^{m} f\left(x_{i}\right)=\frac{\sum_{i=1}^{m} f\left(m x_{i}+\sum_{j=1, j \neq i}^{m} x_{j}\right)+f\left(\sum_{i=1}^{m} x_{i}\right)}{2 m}
$$

where $m$ is a positive integer greater than 2, on fuzzy Banach algebras.

\section{Main results}

We start our work with the following theorem which can be regard as a general solution of functional Equation (1.4).

Theorem 2.1. Let $V$ and $W$ be real vector spaces. A mapping $f: V \rightarrow W$ satisfies in (1.4) if and only if $f$ is additive.

Proof. Setting $x_{j}=0$ in (1.4) $(1 \leq j \leq m)$, we obtain

$$
(m+1) f(0)=2 m^{2} f(0) .
$$

Since $m \geq 2$, we have

$$
f(0)=0 \text {. }
$$

Setting $x_{1}=x, x_{j}=0(2 \leq j \leq m)$ in (1.1), we obtain

$$
f(m x)=m f(x) .
$$

Putting $x_{1}=x, x_{2}=y, x_{j}=0(3 \leq j \leq m)$, we get

$$
f(m x+y)+f(m y+x)+(m-1) f(x+y)=2 m(f(x)+f(y)) .
$$

Putting $x_{1}=x, x_{j}=\frac{y}{m-1}(2 \leq j \leq m)$, we get

$$
f(m x+y)+(m-1) f(2 y+x)+f(x+y)=2 m\left(f(x)+(m-1) f\left(\frac{y}{m-1}\right)\right) .
$$


Let $x=0$ in (2.5), we obtain

$$
2 m(m-1) f\left(\frac{\gamma}{m-1}\right)=2 f(\gamma)+(m-1) f(2 \gamma) .
$$

So, (2.5) turns to following

$$
\begin{aligned}
& f(m x+y)-(m-1) f(2 y+x)+(m-2) f(x+y) \\
& =(2 m-2) f(y)-(m-1) f(2 \gamma) .
\end{aligned}
$$

From (2.4) and (2.7), we have

$$
\begin{aligned}
& f(m x+y)-(m-1) f(2 y+x)+f(x+y) \\
& =2 m f(x)+2 f(y)+(m-1) f(2 y) .
\end{aligned}
$$

Replacing $x$ by $y$ and $y$ by $x$ in (2.7) and comparing it with (2.8), we get

$$
\begin{aligned}
& (m-1)[f(2 x+y)+f(2 y+x)]-(m-3) f(x+y) \\
& =2[f(x)+f(y)]+(m-1)[f(2 x)+f(2 y)] .
\end{aligned}
$$

Letting $x=y$ in (2.4), (2.7), (2.9), respectively, we obtain

$$
\begin{aligned}
& 2 f((m+1) x)+(m-1) f(2 x)=4 m f(x), \\
& f((m+1) x)+(m-1) f(3 x)=(2 m+2) f(x)+(m-2) f(2 x), \\
& f(3 x)=f(2 x)+f(x) .
\end{aligned}
$$

From (2.10)-(2.12) we have

$$
f(2 x)=2 f(x) .
$$

Replacing $f(2 x)$ and $f(2 y)$ by their equivalents by using (2.13) in (2.9), we get

$$
(m-1)[f(2 x+y)+f(2 y+x)]-(m-3) f(x+y)=2 m f(x+y) .
$$

Replacing $y$ by $-x$ in (2.14), we get

$$
f(x)=-f(x) .
$$

Replacing $x$ by $x-y$ in (2.14), we get

$$
(m-1)[f(2 x-y)+f(x+y)]-(m-3) f(x)=2 m(f(x-y)+f(y)) .
$$

Similarly, replacing $y$ by $y-x$ in (2.14), we obtain

$$
(m-1)[f(2 y-x)+f(x+y)]-(m-3) f(y)=2 m(f(x)+f(y-x)) .
$$

Replacing $y$ by $-y$ and $x$ by $-x$ in (2.16) and (2.17), respectively, we obtain

$$
\begin{aligned}
& (m-1)[f(2 x+y)+f(x-y)]-(m-3) f(x)=2 m(f(x+y)+f(-y)), \\
& (m-1)[f(2 y+x)+f(y-x)]-(m-3) f(y)=2 m(f(x+y)+f(-x)) .
\end{aligned}
$$

Adding both sides of (2.18) and (2.19) and using (2.15), we get

$$
(m-1)[f(2 x+y)+f(2 y+x)]+(m+3)[f(x)+f(y)]=4 m f(x+y) .
$$


Comparing (2.20) and (2.14), we obtain $f(x+y)=f(x)+f(y)$ for all $x, y \in V$. So, if a mapping $f$ satisfying (1.4) it must be additive. Conversely, let $f: V \rightarrow W$ be additive, it is clear that $f$ satisfying (1.4) and the proof is complete. In this section we investigate the fuzzy stability of Jordan homomorphisms.

Theorem 2.2. Suppose $(A, N)$ and $(B, N)$ are two fuzzy Banach algebras and $\left(C, N^{p}\right)$ be a fuzzy normed space. Let $\phi: A^{m} \rightarrow C$ be a function such that for some $0<\alpha<m$,

$$
N^{\prime}\left(\varphi\left(a_{1}, \ldots, a_{m}\right), t\right) \geq N^{\prime}\left(\alpha \varphi\left(\frac{a_{1}}{m}, \ldots, \frac{a_{m}}{m}\right), t\right)
$$

for all $a_{1}, \ldots, a_{m} \in A$ and all $t>0$. If $f: A \rightarrow B$ is a mapping such that

$$
N\left(\sum_{i=1}^{m} f\left(a_{i}\right)-\frac{\sum_{i=1}^{m} f\left(m a_{i}+\sum_{j=1, j \neq i}^{m} a_{j}\right)+f\left(\sum_{i=1}^{m} a_{j}\right)}{2 m}, t\right) \geq N^{\prime}\left(\varphi\left(a_{1}, \ldots, a_{m}\right), t\right)
$$

and

$$
N\left(f\left(a^{2}\right)-f(a)^{2}, s\right) \geq N^{\prime}(\varphi(a, \ldots, a), s)
$$

for all $a_{1}, \ldots, a_{m} \in A$ and all $t, s>0$. Then there exists a unique Jordan homomorphism h: $A \rightarrow B$ such that

$$
N(f(a)-h(a), t) \geq N^{\prime}(\varphi(a, 0, \ldots, 0),(m-\alpha) t)
$$

where $a \in A$ and $t>0$.

Proof. Letting $a_{1}=a$ and $a_{2}=\cdots=a_{m}=0$ in (2.22), we obtain

$$
N\left(m^{-1} f(m a)-f(a), m^{-1} t\right) \geq N^{\prime}(\varphi(a, 0, \ldots, 0), t)
$$

for all $a \in A$ and all $t>0$. Replacing $a$ by $m^{j} a$ in (2.25), we have

$$
\begin{aligned}
N\left(m^{-j-1} f\left(m^{j+1} a\right)-m^{-j} f\left(m^{j} a\right), m^{-j-1} t\right) & \geq N^{\prime}\left(\varphi\left(m^{j} a, 0, \ldots, 0\right), t\right) \\
& \geq N^{\prime}\left(\varphi(a, 0, \ldots, 0), \alpha^{-j} t\right)
\end{aligned}
$$

for all $a \in A$, all $t>0$ and any integer $j \geq 0$. So

$$
\begin{aligned}
& N\left(f(x)-m^{-n} f\left(m^{n} a\right), \sum_{j=0}^{n-1} m^{-j-1} \alpha^{j} t\right) \\
& =N\left(\sum_{j=0}^{n-1}\left[m^{-j-1}\left(m^{j+1} a\right)-m^{-j} f\left(m^{j} a\right)\right], \sum_{j=0}^{n-1} m^{-j-1} \alpha^{j} t\right) \\
& \geq \min _{0 \leq j \leq n-1}\left\{N\left(m^{-j-1} f\left(m^{j+1} a\right)-m^{-j} f\left(m^{j} a\right), m^{-j-1} \alpha^{j} t\right)\right\} \\
& \geq N^{\prime}(\varphi(a, 0, \ldots, 0), t)
\end{aligned}
$$

which yields

$$
\begin{aligned}
N\left(m^{-n-p} f\left(m^{n+p} a\right)-m^{-p} f\left(m^{p} a\right), \sum_{j=0}^{n-1} m^{-j-p-1} \alpha^{j} t\right) & \geq N^{\prime}\left(\varphi\left(m^{p} a, 0, \ldots, 0\right), t\right) \\
& \geq N^{\prime}\left(\varphi(a, 0, \ldots, 0), \alpha^{-p} t\right)
\end{aligned}
$$


for all $a \in A, t>0$ and any integers $n>0, p \geq 0$. So

$$
N\left(m^{-n-p} f\left(m^{n+p} a\right)-m^{-p} f\left(m^{p} a\right), \sum_{j=0}^{n-1} m^{-j-p-1} \alpha^{j+p} t\right) \geq N^{\prime}(\varphi(a, 0, \ldots, 0), t)
$$

for all $a \in A, t>0$ and any integers $n>0, p \geq 0$. Hence one obtains

$$
N\left(m^{-n-p} f\left(m^{n+p} a\right)-m^{-p} f\left(m^{p} a\right), t\right) \geq N^{\prime}\left(\varphi(a, 0, \ldots, 0), \frac{t}{\sum_{j=0}^{n-1} m^{-j-p-1} \alpha^{j+p}}\right)
$$

for all $x \in X, t>0$ and any integers $n>0, p \geq 0$. Since, the series $\sum_{j=0}^{+\infty} m^{-j} \alpha^{j}$ is convergent series, we see by taking the limit $p \rightarrow \infty$ in the last inequality that a sequence $\left\{\frac{f\left(m^{n} a\right)}{m^{n}}\right\}$ is a Cauchy sequence in the fuzzy Banach algebra $(B, N)$ and so it converges in $B$. Therefore a mapping $h: A \rightarrow B$ defined by $h(a):=N-\lim _{n \rightarrow \infty} \frac{f\left(m^{n} a\right)}{m^{n}}$ is well defined for all $a \in A$. It means that

$$
\lim _{n \rightarrow \infty} N\left(h(a)-m^{-n} f\left(m^{n} a\right), t\right)=1
$$

for all $a \in A$ and all $t>0$. In addition, it follows from (2.28) that

$$
N\left(f(x)-m^{-n} f\left(m^{n} a\right), t\right) \geq N^{\prime}\left(\sum_{j=0}^{n-1} m^{-j-1} \alpha^{j} \varphi(a, 0, \ldots, 0), t\right)=N^{\prime}\left(\varphi(a, 0, \ldots, 0), \frac{t}{\sum_{j=0}^{n-1} m^{-j-1 \alpha^{j}}}\right)
$$

for all $a \in A$ and all $t>0$. So

$$
\begin{aligned}
N(f(a)-h(a), t) & \geq \min \left\{N\left(f(a)-m^{-n} f\left(m^{n} a\right),(1-\epsilon) t\right), N\left(h(a)-m^{-n} f\left(m^{n} a\right), \epsilon t\right)\right\} \\
& \geq N^{\prime}\left(\sum_{j=0}^{n-1} m^{-j-1} \alpha^{j} \varphi(a, 0, \ldots, 0), t\right)=N^{\prime}\left(\varphi(a, 0, \ldots, 0), \frac{t}{\sum_{j=0}^{n-1} m^{-j-1} \alpha^{j}}\right) \\
& \geq N^{\prime}(\varphi(a, 0, \ldots, 0),(m-\alpha) \epsilon t)
\end{aligned}
$$

for sufficiently large $n$ and for all $a \in A, t>0$ and $\varepsilon$ with $0<\varepsilon<1$. Since $\varepsilon$ is arbitrary and $N^{p}$ is left continuous, we obtain $N(f(a)-h(a), t) \geq N^{p}(\phi(a, 0, \ldots, 0),(m-\alpha) t)$ for all $a \in A$ and $t>0$. It follows from (2.21) and (2.22) that

$$
\begin{aligned}
& N\left(\frac{1}{m^{n}} \sum_{i=1}^{m} f\left(m^{n} a_{i}\right)-\frac{\sum_{i=1}^{m} f\left(m^{n+1} a_{i}+\sum_{j=1, j \neq i}^{m} m^{n} a_{j}\right)+f\left(\sum_{i=1}^{m} m^{n} a_{i}\right)}{2 m^{n+1}}, t\right) \\
& \geq N^{\prime}\left(\varphi\left(m^{n} a_{1}, \ldots, m^{n} a_{m}\right), m^{n} t\right) \geq N^{\prime}\left(\varphi\left(a_{1}, \ldots, a_{m}\right), m^{n} \alpha^{-n} t\right)
\end{aligned}
$$

for all $a_{1}, \ldots, a_{m} \in A, t>0$ and all $n \in \mathbb{N}$. Since $\lim _{n \rightarrow \infty} N^{\prime}\left(\phi\left(a_{1}, \ldots, a_{m}\right), m^{n} \alpha^{-n} t\right)$ $=1$ and so

$$
N\left(\frac{1}{m^{n}} \sum_{i=1}^{m} f\left(m^{n} a_{i}\right)-\frac{\sum_{i=1}^{m} f\left(m^{n+1} a_{i}+\sum_{j=1, j \neq i}^{m} m^{n} a_{j}\right)+f\left(\sum_{i=1}^{m} m^{n} a_{i}\right)}{2 m^{n+1}}, t\right) \rightarrow 1
$$


for all $a_{1}, \ldots, a_{m} \in A$ and all $t>0$. Therefore, we obtain in view of (2.29)

$$
\begin{aligned}
& N\left(\sum_{i=1}^{m} h\left(a_{i}\right)-\frac{\sum_{i=1}^{m} h\left(m a_{i}+\sum_{j=1, j \neq i}^{m} a_{j}\right)+h\left(\sum_{i=1}^{m} a_{i}\right)}{2 m}, t\right) \\
& \geq \min \left\{N \left(\sum_{i=1}^{m} h\left(a_{i}\right)-\frac{\sum_{i=1}^{m} h\left(m a_{i}+\sum_{j=1, j \neq i}^{m} a_{j}\right)+h\left(\sum_{i=1}^{m} a_{i}\right)}{2 m}\right.\right. \\
& \left.-\frac{1}{m^{n}} \sum_{i=1}^{m} f\left(m^{n} a_{i}\right)-\frac{\sum_{i=1}^{m} f\left(m^{n+1} a_{i}+\sum_{j=1, j \neq i}^{m} m^{n} a_{j}\right)+f\left(\sum_{i=1}^{m} m^{n} a_{i}\right)}{2 m^{n+1}}, \frac{t}{2}\right) \\
& \left., N\left(\frac{1}{m^{n}} \sum_{i=1}^{m} f\left(m^{n} a_{i}\right)-\frac{\sum_{i=1}^{m} f\left(m^{n+1} a_{i}+\sum_{j=1, j \neq i}^{m} m^{n} a_{j}\right)+f\left(\sum_{i=1}^{m} m^{n} a_{i}\right)}{2 m^{n+1}}, \frac{t}{2}\right)\right\} \\
& =N\left(\frac{1}{m^{n}} \sum_{i=1}^{m} f\left(m^{n} a_{i}\right)-\frac{\sum_{i=1}^{m} f\left(m^{n+1} a_{i}+\sum_{j=1, j \neq i}^{m} m^{n} a_{j}\right)+f\left(\sum_{i=1}^{m} m^{n} a_{i}\right)}{2 m^{n+1}}, \frac{t}{2}\right) \\
& \geq N^{\prime}\left(\varphi\left(a_{1}, \ldots, a_{m}\right), \frac{m^{n} \alpha^{-n} t}{2}\right) \rightarrow 1 \text { as } n \rightarrow \infty
\end{aligned}
$$

which implies $\sum_{i=1}^{m} h\left(a_{i}\right)=\frac{\sum_{i=1}^{m} h\left(m a_{i}+\sum_{j=1, j \neq i}^{m} a_{j}\right)+h\left(\sum_{i=1}^{m} a_{i}\right)}{2 m}$ for all $a_{1}, \ldots, a_{m} \in A$. Thus $h: A \rightarrow B$ is a mapping satisfying the Equation (1.4) and the inequality (2.24). To prove the uniqueness, let there is another mapping $k: A \rightarrow B$ which satisfies the inequality (2.24).

Then, for all $a \in A$, we have

$$
\begin{aligned}
N(h(a)-k(a), t)= & N\left(m^{-n} h\left(m^{n} a\right)-m^{-n} k\left(m^{n} a\right), t\right) \\
\geq & \min \left\{N\left(m^{-n} h\left(m^{n} a\right)-m^{-n} f\left(m^{n} a\right), \frac{t}{2}\right),\right. \\
& \left.N\left(m^{-n} f\left(m^{n} a\right)-m^{-n} k\left(m^{n} a\right), \frac{t}{2}\right)\right\} \\
\geq & N^{\prime}\left(\varphi\left(m^{n} a, 0, \ldots, 0\right), \frac{m^{n}(m-\alpha) t}{2}\right) \\
\geq & N^{\prime}\left(\varphi(a, 0, \ldots, 0), \frac{m^{n}(m-\alpha) t}{2 \alpha^{n}}\right) \rightarrow 1 \text { as } n \rightarrow \infty
\end{aligned}
$$

for all $t>0$. Therefore $h(a)=k(a)$ for all $a \in A$. Now we only need to show that $h$ $\left(a^{2}\right)=h(a)^{2}$ for all $a \in A$. It follows from (2.24) that

$$
N\left(f\left(m^{n} a\right)-h\left(m^{n} a\right), t\right) \geq N^{\prime}\left(\frac{\varphi\left(m^{n} a, 0, \ldots, 0\right)}{m-\alpha}, t\right) \geq N^{\prime}\left(\varphi(a, 0, \ldots, 0), \frac{(m-\alpha) t}{\alpha^{n}}\right)
$$

for all $a \in A$ and all $t>0$. Thus $N\left(m^{-n} f\left(m^{n} a\right)-m^{-n} h\left(m^{n} a\right), m^{-n} t\right) \geq N^{\prime}\left(\varphi(a, 0, \ldots, 0), \frac{(m-\alpha) t}{\alpha^{n}}\right)$ for all $a \in A$ and all $t>0$. By the additivity of $h$ it is easy to see that

$$
N\left(m^{-n} f\left(m^{n} a\right)-h(a), t\right) \geq N^{\prime}\left(\varphi(a, 0, \ldots, 0), \frac{m^{n}(m-\alpha) t}{\alpha^{n}}\right)
$$

for all $a \in A$ and all $t>0$. Letting $n$ to infinity in (2.30) and using $\left(N_{5}\right)$, we see that

$$
h(a)=N-\lim _{n \rightarrow \infty} m^{-n} f\left(m^{n} a\right), \quad \text { and } \quad h\left(a^{2}\right)=N-\lim _{n \rightarrow \infty} m^{-2 n} f\left(m^{2 n} a^{2}\right)
$$


for all $a \in A$. Using inequality (2.23), we get

$$
N\left(f\left(m^{2 n} a^{2}\right)-f\left(m^{n} a\right)^{2}, s\right) \geq N^{\prime}\left(\varphi\left(m^{n} a, \ldots, m^{n} a\right), s\right) \geq N^{\prime}\left(\alpha^{n} \varphi(a, \ldots, a), s\right)
$$

for all $a \in A$ and all $s>0$. Thus

$$
N\left(\frac{f\left(m^{2 n} a^{2}\right)-f\left(m^{n} a\right)^{2}}{m^{2 n}}, s\right) \geq N^{\prime}\left(\varphi(a, \ldots, a), \frac{m^{2 n} s}{\alpha^{n}}\right)
$$

for all $a, b \in A$ and all $s>0$. Letting $n$ to infinity in (2.32) and using $\left(N_{5}\right)$, we see that

$$
N-\lim _{n \rightarrow \infty} \frac{f\left(m^{2 n} a^{2}\right)-f\left(m^{n} a\right)^{2}}{m^{2 n}}=0 .
$$

Applying (2.31) and (2.33), we have

$$
\begin{aligned}
h\left(a^{2}\right)=N-\lim _{n \rightarrow \infty} \frac{f\left(m^{2 n} a^{2}\right)-f\left(m^{n} a\right)^{2}}{m^{2 n}} & =N-\lim _{n \rightarrow \infty} \frac{f\left(m^{2 n} a^{2}\right)-f\left(m^{2 n} a^{2}\right)+f\left(m^{n} a\right)^{2}}{m^{2 n}} \\
& =N-\lim _{n \rightarrow \infty} \frac{f\left(m^{n} a\right)^{2}}{m^{2 n}}=\left[N-\lim _{n \rightarrow \infty} \frac{f\left(m^{n} a\right)}{m^{n}}\right]^{2} \\
& =h(a)^{2}
\end{aligned}
$$

for all $a \in A$. To prove the uniqueness of $h$, assume that $h^{\prime}$ is another Jordan homomorphism satisfying (2.24). Since both $h$ and $h$ ' are additive, we deduce that

$$
\begin{aligned}
N\left(h(a)-h^{\prime}(a), t\right)=N\left(h\left(m^{n} a\right)-h^{\prime}\left(m^{n} a\right), m^{n} t\right) & \geq N^{\prime}\left(\frac{\varphi\left(m^{n} a, 0, \ldots, 0\right)}{m-\alpha}, \frac{m^{n} t}{2}\right) \\
& \geq N^{\prime}\left(\varphi(a, 0, \ldots, 0), \frac{m^{n}(m-\alpha) t}{2 \alpha^{n}}\right)
\end{aligned}
$$

for all $a \in A$ and all $t>0$. Letting $n$ to infinity, we infer that $N\left(h(a)-h^{\prime}(a), t\right)=1$ for all $a \in A$ and all $t>0$. Hence $\left(N_{2}\right)$ implies that $h(a)=h^{\prime}(a)$ for all $a \in A$. $\square$

Corollary 2.3. Suppose $(A, N)$ and $(B, N)$ are two fuzzy Banach algebras and $\left(C, N^{\prime}\right)$ be a fuzzy normed space. If $f: A \rightarrow B$ is a mapping such that

$$
N\left(\sum_{i=1}^{m} f\left(a_{i}\right)-\frac{\sum_{i=1}^{m} f\left(m a_{i}+\sum_{j=1, j \neq i}^{m} a_{j}\right)+f\left(\sum_{i=1}^{m} a_{i}\right)}{2 m}, t\right) \geq N^{\prime}\left(\theta \sum_{i=1}^{n}\left\|a_{i}\right\|^{r}, t\right)
$$

and

$$
N\left(f\left(a^{2}\right)-f(a)^{2}, s\right) \geq N^{\prime}\left(n \theta\|a\|^{r}, s\right)
$$

for all $a_{1}, \ldots, a_{n}, a \in A$ and all $t, s>0$. Then there exists a unique Jordan homomorphism h: $A \rightarrow B$ such that

$$
N(f(a)-h(a), t) \geq N^{\prime}\left(\theta\|a\|^{r},(m-1) t\right)
$$

where $a \in A$ and $t>0$.

Proof. Letting $\varphi\left(a_{1}, \ldots, a_{n}\right)=\theta \sum_{i=1}^{n}\left\|a_{i}\right\|^{r}$ and $\alpha=1$. Applying Theorem 2.2, we obtain the desired results. $\square$

\section{Fuzzy stability of Jordan derivations}

In this section we prove the stability of Jordan derivations on fuzzy Banach algebras. 
Theorem 3.1. Let $(A, N)$ be a fuzzy Banach algebra and $\left(B, N^{\prime}\right)$ be a fuzzy normed space.

Let $\phi: A^{m} \rightarrow B$ be a function such that for some $0<\alpha<m$,

$$
N^{\prime}\left(\varphi\left(a_{1}, \ldots, a_{m}\right), t\right) \geq N^{\prime}\left(\alpha \varphi\left(\frac{a_{1}}{m}, \ldots, \frac{a_{m}}{m}\right), t\right)
$$

for all $a, b \in A$ and all $t>0$. Suppose that $f: A \rightarrow A$ is a function such that

$$
N\left(\sum_{i=1}^{m} f\left(a_{i}\right)-\frac{\sum_{i=1}^{m} f\left(m a_{i}+\sum_{j=1, j \neq i}^{m} a_{j}\right)+f\left(\sum_{i=1}^{m} a_{i}\right)}{2 m}, t\right) \geq N^{\prime}\left(\varphi\left(a_{1}, \ldots, a_{m}\right), t\right)
$$

and

$$
N\left(f\left(a^{2}\right)-a f(a)-f(a) a, s\right) \geq N^{\prime}(\varphi(a, \ldots, a), s)
$$

for all $a, b \in A$ and all $t, s>0$. Then there exists a unique Jordan derivation $d: A \rightarrow$ A such that

$$
N(f(a)-d(a), t) \geq N^{\prime}(\varphi(a, 0, \ldots, a),(m-\alpha) t)
$$

where $a \in A$ and $t>0$.

Proof. Proceeding as in the proof of Theorem 2.2, we find that there exists an additive function $d: A \rightarrow A$ satisfying (3.4). Now we only need to show that $d$ satisfies $d$ $\left(a^{2}\right)=a d(a)+d(a) a$ for all $a \in A$. The inequalities (3.1) and (3.4) imply that

$$
N\left(f\left(m^{n} a\right)-d\left(m^{n} a\right), t\right) \geq N^{\prime}\left(\frac{\varphi\left(m^{n} a, 0, \ldots, 0\right)}{m-\alpha}, t\right) \geq N^{\prime}\left(\varphi(a, 0, \ldots, 0), \frac{(m-\alpha) t}{\alpha^{n}}\right)
$$

for all $a \in A$ and all $t>0$. Thus

$$
N\left(m^{-n} f\left(m^{n} a\right)-m^{-n} d\left(m^{n} a\right), m^{-n} t\right) \geq N^{\prime}\left(\varphi(a, 0, \ldots, 0), \frac{(m-\alpha) t}{\alpha^{n}}\right)
$$

for all $a \in A$ and all $t>0$. By the additivity of $d$ it is easy to see that

$$
N\left(m^{-n} f\left(m^{n} a\right)-d(a), t\right) \geq N^{\prime}\left(\varphi(a, 0, \ldots, 0), \frac{m^{n}(m-\alpha) t}{\alpha^{n}}\right)
$$

for all $a \in A$ and all $t>0$. Letting $n$ to infinity in (3.5) and using $\left(N_{5}\right)$, we get

$$
d(a)=N-\lim _{n \rightarrow \infty} m^{-n} f\left(m^{n} a\right) \quad \text { and } \quad d\left(a^{2}\right)=N-\lim _{n \rightarrow \infty} m^{-2 n} f\left(m^{2 n} a^{2}\right)
$$

for all $a \in A$. Using (3.1) and (3.3), we get

$$
\begin{aligned}
N\left(f\left(m^{2 n} a^{2}\right)-\left(m^{n} a\right) f\left(m^{n} a\right)-f\left(m^{n} a\right)\left(m^{n} a\right), s\right) & \geq N^{\prime}\left(\varphi\left(m^{n} a, 0, \ldots, 0\right), s\right) \\
& \geq N^{\prime}\left(\varphi(a, 0, \ldots, 0), \frac{s}{\alpha^{n}}\right)
\end{aligned}
$$

for all $a \in A$ and all $s>0$. Let $g: A \times A \rightarrow A$ be a function defined by $g(a, a)=f\left(a^{2}\right)$ af(a) - $f(a) a$ for all $a \in A$. Hence, (3.7) implies that

$$
N-\lim _{n \rightarrow \infty} m^{-n} g\left(m^{n} a, m^{n} a\right)=0, \quad \text { and } \quad N-\lim _{n \rightarrow \infty} m^{-2 n} g\left(m^{n} a, m^{n} a\right)=0
$$


for all $a \in A$. Since $(A, N)$ is a fuzzy Banach algebra, applying (3.6) and (3.8), we get

$$
\begin{aligned}
d\left(a^{2}\right) & =N-\lim _{n \rightarrow \infty} m^{-2 n} f\left(m^{2 n} a^{2}\right) \\
& =N-\lim _{n \rightarrow \infty}\left[a m^{-n} f\left(m^{n} a\right)+m^{-n} f\left(m^{n} a\right) a+m^{-2 n} g\left(m^{n} a, m^{n} a\right)\right] \\
& =a\left(N-\lim _{n \rightarrow \infty} m^{-n} f\left(m^{n} a\right)\right)+\left(N-\lim _{n \rightarrow \infty} m^{-n} f\left(m^{n} a\right)\right) a+N-\lim _{n \rightarrow \infty} m^{-2 n} g\left(m^{n} a, m^{n} a\right) \\
& =a d(a)+d(a) a .
\end{aligned}
$$

for all $a \in A$. To prove the uniqueness property of $d$, assume that $d$ ' is another Jordan derivation satisfying (3.4). Since both $d$ and $d^{\prime}$ are additive we deduce that

$$
\begin{aligned}
N\left(d(a)-d^{\prime}(a), t\right)=N\left(d\left(m^{n} a\right)-d^{\prime}\left(m^{n} a\right), m^{n} t\right) & \geq N^{\prime}\left(\varphi\left(m^{n} a, 0, \ldots, 0\right), \frac{m^{n}(m-\alpha) t}{2}\right) \\
& \geq N^{\prime}\left(\varphi(a, 0, \ldots, 0), \frac{m^{n}(m-\alpha) t}{2 \alpha^{n}}\right)
\end{aligned}
$$

for all $a \in A$ and all $t>0$. Letting $n$ to infinity in the above inequality, we get $N(d(a)$ - $\left.d^{\prime}(a), t\right)=1$ for all $a \in A$ and all $t>0$. Hence $d(a)=d^{\prime}(a)$ for all $a \in A$.

Corollary 3.2. Suppose $(A, N)$ and $(B, N)$ are two fuzzy Banach algebras and $\left(C, N^{r}\right)$ be a fuzzy normed space. If $f: A \rightarrow B$ is a mapping such that

$$
N\left(\sum_{i=1}^{m} f\left(a_{i}\right)-\frac{\sum_{i=1}^{m} f\left(m a_{i}+\sum_{j=1, j \neq i}^{m} a_{j}\right)+f\left(\sum_{i=1}^{m} a_{i}\right)}{2 m}, t\right) \geq N^{\prime}\left(\theta \sum_{i=1}^{n}\left\|a_{i}\right\|^{r}, t\right)
$$

and

$$
N\left(f\left(a^{2}\right)-f(a)^{2}, s\right) \geq N^{\prime}\left(n \theta\|a\|^{r}, s\right)
$$

for all $a_{1}, \ldots, a_{n}, a \in A$ and all $t, s>0$. Then there exists a unique Jordan homomorphism h: $A \rightarrow B$ such that

$$
N(f(a)-h(a), t) \geq N^{\prime}\left(\theta\|a\|^{r}, \frac{\left(m^{2}-1\right) t}{m}\right)
$$

where $a \in A$ and $t>0$.

Proof. Letting $\varphi\left(a_{1}, \ldots, a_{n}\right)=\theta \sum_{i=1}^{n}\left\|a_{i}\right\|^{r}$ and $\alpha=\frac{1}{m}$. Applying Theorem 3.1, we get the desired results. $\square$

\section{Conclusion}

We establish the generalized Hyers-Ulam stability of Jordan homomorphisms and Jordan derivations on fuzzy Banach algebras. We show that every approximately Jordan homomorphism (Jordan derivation) is near to an exact Jordan homomorphism (Jordan derivation).

\section{Acknowledgements}

Dong Yun Shin was supported by the Basic Science Research Program through the National Research Foundation of Korea funded by the Ministry of Education, Science and Technology (NRF-2010-0021792).

\section{Author details}

'Department of Mathematics, Yasouj University, Yasouj 75914-353, Iran ${ }^{2}$ Department of mathematics, Islamic Azad University, Bushehr Branch, Bushehr, Iran ${ }^{3}$ Department of Mathematics, Semnan University, P. O. Box 35195-363, Semnan, Iran ${ }^{4}$ Department of Mathematics, College of Natural Science, University of Seoul, Seoul 130-743, Korea 


\section{Authors' contributions}

All authors conceived of the study participated in its design and coordination, drafted the manuscript, participated in the sequence alignment, and read and approved the final manuscript.

\section{Competing interests}

The authors declare that they have no competing interests.

Received: 29 February 2012 Accepted: 29 May 2012 Published: 29 May 2012

\section{References}

1. Ulam, SM: A Collection of the Mathematical Problems. Interscience Publ., New York (1960)

2. Hyers, DH: On the stability of the linear functional equation. Proc Natl Acad Sci. 27, 222-224 (1941). doi:10.1073/ pnas.27.4.222

3. Rassias, ThM: On the stability of the linear mapping in Banach spaces. Proc Am Math Soc. 72, 297-300 (1978). doi:10.1090/S0002-9939-1978-0507327-1

4. Gajda, Z: On stability of additive mappings. Int J Math Math Sci. 14, 431-434 (1991). doi:10.1155/S016117129100056X

5. Bag, T, Samanta, SK: Finite dimensional fuzzy normed linear spaces. J Fuzzy Math. 11(3), 687-705 (2003)

6. Eshaghi Gordji, M, Khodaei, $\mathrm{H}$ : The fixed point method for fuzzy approximation of a functional equation associated with inner product spaces. Discr Dyn Nature Soc 2010, 15 (2010). (Article ID 140767). doi:10.1155/2010/140767

7. Ghobadipour, N, Park, C: Cubic-quartic functional equations in fuzzy normed spaces. Int J Nonlinear Anal Appl. 1, 12-21 (2010)

8. Khodaei, H, Kamyar, M: Fuzzy approximately additive mappings. Int J Nonlinear Anal Appl. 1(2), 44-53 (2010)

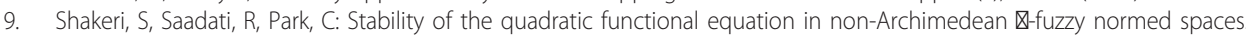
Int J Nonlinear Anal Appl. 1(2), 72-83 (2010)

10. Bavand Savadkouhi, M, Gordji, ME, Rassias, JM, Ghobadipour, N: Approximate ternary Jordan derivations on Banach ternary algebras. J Math Phys. 50, 042303 (2009). doi:10.1063/1.3093269

11. Cho, YJ, Kang, Jl, Saadati, R: Fixed points and stability of additive functional equations on the Banach algebras. J Comput Anal Appl. 14, 1103-1111 (2012)

12. Ebadian, A, Ghobadipour, N, Gordji, ME: A fixed point method for perturbation of bimultipliers and Jordan bimultipliers in $C^{*}$-ternary algebras. J Math Phys 51(1), 10 (2010). doi:10.1063/1.3496391

13. Eshaghi Gordji, M, Ghaemi, MB, Kaboli Gharetapeh, S, Shams, S, Ebadian, A: On the stability of J*-derivations. J Geom Phys. 60(3), 454-459 (2010). doi:10.1016/j.geomphys.2009.11.004

14. Eshaghi Gordji, M, Kaboli Gharetapeh, S, Karimi, T, Rashidi, E, Aghaei, M: Ternary Jordan derivations on $C^{*}$-ternary algebras. J Comput Anal Appl. 12(2), 463-470 (2010)

15. Eshaghi Gordji, M, Karimi, T, Kaboli Gharetapeh, S: Approximately n-Jordan homomorphisms on Banach algebras. J Ineq Appl 2009, 8 (2009). (Article ID 870843)

16. Eshaghi Gordji, M, Najati, A: Approximately J*-homomorphisms: a fixed point approach. J Geom Phys. 60, 809-814 (2010). doi:10.1016/j.geomphys.2010.01.012

17. Eshaghi Gordji, M, Rassias, JM, Ghobadipour, N: Generalized Hyers-Ulam stability of the generalized ( $n, k)$-derivations. Abstr Appl Anal 2009, 8 (2009). (Article ID 437931)

18. Farokhzad, R, Hosseinioun, SAR: Perturbations of Jordan higher derivations in Banach ternary algebras: an alternative fixed point approach. Int J Nonlinear Anal Appl. 1(1), 42-53 (2010)

19. Najati, A, Kang, Jl, Cho, YJ: Local stability of the pexiderized Cauchy and Jensen's equations in fuzzy Spaces. J Inequal Appl. 2011, 78 (2011). doi:10.1186/1029-242X-2011-78

20. Park, C, Eshaghi Gordji, M: Comment on "Approximate ternary Jordan derivations on Banach ternary algebras". J Math Phys 51, 044102 (2010). doi:10.1063/1.3299295. [Bavand Savadkouhi et al. J. Math. Phys. 50, 042303 (2009)]. doi:10.1063/ 1.3299295

21. Park, C, Najati, A: Generalized additive functional inequalities in Banach algebras. Int J Nonlinear Anal Appl. 1(2), 54-62 (2010)

22. Rassias, ThM: Functional Equations, Inequalities and Applications. Kluwer Academic Publishers Company, Dordrecht (2003)

23. Rassias, ThM: Problem 16;2, Report of the 27th international symp. on functional equations Aequations Math. 39, 292-293 (1990)

24. Rassias, ThM: On the stability of the quadratic functional equation and its applications. Studia Univ Babes-Bolyai. XLIII, 89-124 (1998)

25. Rassias, ThM: The problem of S.M. Ulam for approximately multiplicative mappings. J Math Anal Appl. 246, 352-378 (2000). doi:10.1006/jmaa.2000.6788

26. Rassias, ThM: On the stability of functional equations in Banach spaces. J Math Anal Appl. 251, 264-284 (2000). doi:10.1006/jmaa.2000.7046

27. Rassias, ThM, Semrl, P: On the behaviour of mappings which do not satisfy Hyers-Ulam stability. Proc Am Math Soc. 114, 989-993 (1992). doi:10.1090/S0002-9939-1992-1059634-1

28. Rassias, ThM, Semrl, P: On the Hyers-Ulam stability of linear mappings. J Math Anal Appl. 173, 325-338 (1993). doi:10.1006/jmaa.1993.1070

29. Saadati, R, Cho, YJ, Vahidi, J: The stability of the quartic functional equation in various spaces. Comput Math Appl. 60 , 1994-2002 (2010). doi:10.1016/j.camwa.2010.07.034

doi:10.1186/1687-1847-2012-70

Cite this article as: Azadi Kenary et al:: Almost Jordan homomorphisms and Jordan derivations associated to the parametric-additive functional equation on fuzzy Banach algebras. Advances in Difference Equations 2012 2012:70. 